\title{
Habituación a largo plazo y claves ambientales en humanos:
}

\author{
J. L. GONZÁLEZ y J. M. GRANDE \\ Universidad Complutense de Madrid
}

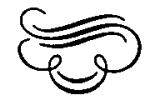

Resumen

Con objeto de comprobar si, como el modelo de Wagner $(1976,1978)$ postula, durante la babituación a largo plazo se establece una asociación entre el estimulo habituador y las claves ambientales, se llevaron a cabo dos experimentos en humanos. En el experimento I el cambio de contexto ambiental produjo una rehabituación más rápida y una tendencia a una menor recuperación de la respuesta en la segunda situación (empleando un tono de 105 dbs., comienzo súbito y veinticuatro boras de intervalo entre sesiones). En el experimento II (empleando un tono de 80 dbs., comienzo gradual y cuarenta y ocho horas de intervalo entre sesiones), el cambio de contexto ambiental produjo una menor recuperación de la respuesta y una tendencia a una mayor tasa de habituación. Las instrucciones sinformativas. no produjeron habituación diferente de las "normales*. Se consideran estos resultados como acordes con una interpretación atencional tradicional de la habituación más que con el modelo de Wagner.

\section{Abstract}

Two experiments with human subjects were carried out in order to verify the role that contextual cues play in long term habituation, acording with the Wagner's $(1976,1978)$ model. Experiment I, employing a $105 \mathrm{dbs}$., tone of sudden onset and 24 hours separation between sessions, showed that contextual change produced faster rebabituation and a trend to less response reconvery in the second session. Experiment $I I$, employing a $80 \mathrm{dbs}$., tone of gradual onset with 48 bours between sessions, showed that contextual change produced less response recovery and a trend to faster rehabituation. "Informative* vs. "normal* instructions did not produce any effect on habituation. These results are considered as contrary to Wagner's (1976, 1978) model and in accordance with an atentional interpretation of habituation.

Dirección de los autores: Universidad Complutense de Madrid. Facultad de Psicología. Campus de Somosaguas. 28025 Madrid.

Agradecimiento: Queremos expresar nuestro agradecimiento al Parque Atracciones (Madrid), en especial al director del mismo don Antonio Rivas, por las facilidades dadas para la realización de estos experimentos. 
Wagner $(1976,1978)$ ha formulado un modelo teórico sobre la habituación según el cual ésta se produce cuando la presentación del estímulo habituador coincide con una prerrepresentación a tal estímulo en la memoria a corto plazo. Dicha prerrepresentación puede estar generada por la reciente presentación del estímulo habituador (autogenerada) o por la exposición a las claves ambientales con las que el estímulo habituador se habría asociado durante los ensayos de habituación (generada por evocación). La prerrepresentación autogenerada sería la responsable de la habituación a corto plazo, mientras que la habituación a largo plazo dependería de la asociación clave ambientales-estímulo habituador.

El mismo Wagner (1976) informa de los resultados de un experimento realizado para verificar esta hipótesis, en el que se estudió la habituación acústica de la respuesta de vasoconstricción en el conejo. Después del entrenamiento en habituación, parte de los sujetos permanecieron en las jaulas y el resto en los aparatos experimentales (es decir, expuesto a las claves ambientales-estímulo habituador previamente adquirida durante la habituación. Tal como cabía esperar, el grupo expuesto a las claves ambientales mostró una recuperación de la respuesta mayor en una prueba de retención posterior.

Existen, sin embargo, algunos estudios, parte de ellos anteriores incluso a la formulación del modelo, cuyos resultados no concuerdan con el mismo. Así, Korn y Moyer (1966) estudiaron la habituación acústica de la respuesta de sobresalto en ratas en función de la preexposición a las claves ambientales. Un grupo de sujetos fue expuesto durante treinta minutos al día a lo largo de una semana a las claves ambientales antes de pasar al entrenamiento en habituación. Otro grupo recibió el entrenamiento en habituación normal, sin preexposición. Según el modelo de Wagner cabría esperar que la preexposición a las claves ambientales solas, dificultase su asociación posterior con el estímulo habituador al producirse así una inhibición latente de dicha asociación, por lo que la habituación debería ser menor. Sin embargo, los sujetos preexpuestos mostraron un decremento en las respuestas mayor que el de los sujetos que no recibieron preexposición.

Peeke y Veno (1973), estudiaron los efectos del cambio en las claves de fondo sobre la habituación, en la segunda se cambió la localización del pez intruso en algunos sujetos, su identidad en otros y ambas cosas en otros. El cambio en la localización del pez intruso puede considerarse como un cambio en las claves ambientales del estímulo habituador. Sin embargo, no se obtuvieron diferencias entre un grupo de control con exposición al estímulo en la misma localización en ambas sesiones y el grupo en que sólo ésta cambió.

Leaton (1974), en una serie de experimentos sobre la retención de la habituación de la supresión del lameteo en ratas, introdujo algunos cambios en las claves ambientales (apagado de la luz y cambio del suelo de rejilla por otro tupido), entre la primera y la segunda sesión. En la segunda sesión no se observó ninguna interrupción del decremento de la respuesta de lameteo en las ratas. El cambio en las claves ambientales no afectó a la habituación a largo plazo.

Marlin y Miller (1981) han realizado una serie de experimentos con el objetivo expreso de verificar el modelo de Wagner en la habituación acústica de la respuesta de sobresalto en ratas. Estos autores intentaron: 1) el 
ensombrecimiento de la hipotética asociación estímulo habituador-claves ambientales mediante la provisión de un predictor del primero más fiable que estas últimas. 2) La deshabituación mediante la introducción de grandes cambios en las claves ambientales (iluminación, textura del suelo, las paredes y el olor) entre la primera y la segunda sesión de habituación. 3) La extinción de la supuesta asociación claves ambientales-estímulo habituador mediante varios tipos de exposición no emparejada a dichos estímulos. 4) La inhibición latente de tal hipotética asociación mediante la preexposición a las claves ambientales solas antes del entrenamiento en habituación. Los resultados de todos estos experimentos fueron homogéneos y contrarios a las predicciones hechas a partir del modelo de Wagner. Tales manipulaciones no produjeron ningún efecto sobre la habituación a largo plazo.

Glez. Almendros (1983), empleando la habituación de la respuesta de sobresaltos en ratas también introdujo cambios muy notables en las claves ambientales (olfativas, visuales, táctiles y posturales) entre la primera y la segunda sesión en habituación en un grupo, cambios parciales en las claves ambientales en otro grupo, cambios en las claves internas (mediante una inyección de cloruro de litio en la primera sesión) en un tercer grupo y ningún cambio en un cuarto grupo. Aunque el cambio en las claves internas afectó a la habituación a largo plazo, los cambios en las claves ambientales no produjeron ningún efecto, ni en la rehabilitación ni en la recuperación de la respuesta en el primer ensayo de la segunda sesión con relación al último de la primera.

Como puede observarse, los estudios realizados con animales no apoyan, salvo el realizado por Wagner (1976), el modelo asociativo de este autor. El único trabajo sobre el tema realizado hasta la fecha con humanos ha sido el de Averill, Malmstrom, Koriat y Lazarus (1972), quienes estudiaron la habituación de varias respuestas (ritmo cardiaco, conductancia de la piel y autoinforme) ante estímulos emocionales complejos en función de su contexto de presentación. El estímulo habituador era la visión de la escena de un accidente en la que un trabajador se amputaba dos falanges de un dedo. El grupo experimental fue sometido a 20 exposiciones de este estímulo durante tres sesiones, mientras que el grupo de control lo era a otras imágenes diferentes. Después de cada sesión a ambos grupos se les exhibió una película completa de doce minutos de duración en la que se incluían tres situaciones de accidente, el segundo de ellos era el estímulo habituador empleado en el grupo experimental. El ritmo cardiaco indicó que la habituación ante el segundo accidente (el estímulo habituador) visionado en solitario se mantuvo para esa misma escena visionada en el contexto de la película completa. Para la conductancia de la piel y los autoinformes, la repetida presentación en solitario de la escena del accidente produjo una marcada reducción en el arousal emocional cuando la escena fue vista dentro de la película completa. Como se puede observar, tampoco estos resultados han sido favorables al modelo de Wagner.

Debido a que este experimento empleó estímulos complejos, es el único con sujetos humanos, de características algo atípicas y realizado antes de su formulación teórica, consideramos que tenía interés extender los estudios de verificación del modelo de Wagner a sujetos humanos. En el experimento I se empleó como estímulo habituador un sonido de comienzo súbito como habitualmente se administra, e intensidad media-alta con ins- 


\section{8}

trucciones neutras y un intervalo de retención de veinticuatro horas. En el segundo, se empleó un estímulo de comienzo gradual e intensidad moderada e instrucciones tanto neutras como informativas y un intervalo de retención de cuarenta y ocho horas. En ambos casos se alteraron drásticamente las claves ambientales en las que dicho estímulo se presentaba entre la primera y la segunda sesión. El objetivo principal era averiguar si el cambio en las claves ambientales afectaba a la habituación a largo plazo de la Respuesta de Resistencia de la Piel (RRP).

\section{EXPERIMENTO I}

De acuerdo con el modelo teórico de Wagner $(1976,1978)$, si la habituación a largo plazo se produce por la asociación del estímulo habituador con las claves ambientales presentes, entonces cabría esperar que al cambiar radicalmente éstas en la segunda sesión de habituación realizada veinticuatro horas después de la primera, la respuesta se recuperará prácticamente en su totalidad y, en cualquier caso, más que si aquéllas no se cambian. Además, en tal caso, la rehabituación es de esperar que sea más lenta y con distinto curso, por tener que establecerse de nuevo.

\section{Método}

\section{Sujetos}

Los sujetos fueron 19 estudiantes de sexo femenino que cursaban estudios de primero en la Facultad de Psicología de la Universidad Complutense de Madrid, y de edades entre 18 y 24 años. Los sujetos participaron voluntariamente en el experimento después de habérseles informado muy vagamente de algunos aspectos de la investigación y con la promesa de una explicación detallada una vez finalizado el experimento. Entre la Sesión I y la Sesión II del experimento (veinticuatro horas de intervalo) se perdió, por inasistencia, un sujeto del grupo experimental y otro del grupo control, que se compusieron definitivamente de 10 y 8 sujetos respectivamente. En el grupo experimental se eliminó después un sujeto que ya conocía la «Casa Magnética» donde se llevó a cabo la Sesión II de este grupo.

\section{Aparatos}

Para la presentación de los tonos se empleó un audiogenerador Lafayette VIZ NA-5048/44D cuya señal era introducida en un amplificador Lafayette 15020 y terminaba en un altavoz situado a un metro del sujeto. La duración del sonido utilizado como estímulo habituador era fijado mediante un temporizador de relé. La Respuesta de Resistencia de la Piel (RRP) se registró mediante un polígrafo Lafayette LA-76100-01. Se midió su amplitud entendida como la deflexión máxima de la curva cuyo comienzo ocurriera entre uno y cuatro segundos después de administrado el tono. La humedad relativa del aire se midió con higrómetro de pelo convencional, y el tiempo con un cronómetro de laboratorio. 
Diseño

El diseño empleado se componía de dos grupos (Grupo E y Grupo C) cuyos sujetos fueron aleatoriamente asignados a cada uno de ellos. Como esquematiza la tabla I, ambos grupos recibieron entrenamiento de habituación en dos sesiones (Sesión I y Sesión II) separadas veinticuatro horas entre sí, pero mientras el Grupo E recibió el entrenamiento de la Sesión II en una situación nueva, diferente a la de la Sesión I (con claves ambientales nuevas), el Grupo C recibió el entrenamiento de ambas sesiones en la misma situación (claves ambientales idénticas).

TABLA I

Diseño empleado en el experimento I

\begin{tabular}{c|c|c|c|}
\multicolumn{1}{c}{} & \multicolumn{2}{c}{ Sesión I } & \multicolumn{1}{c}{$\begin{array}{c}\text { Variable } \\
\text { independiente }\end{array}$} \\
\cline { 2 - 4 } Grupo E & Situación A & Situación B & Clases ambientales nuevas \\
\hline \multirow{2}{*}{ Grupo C } & Situación A & Situación A & Clases ambientales idénticas \\
\hline
\end{tabular}

En ausencia de otras alteraciones diferencias entre ambos grupos, las diferencias en la rehabituación de la RRP entre ellos serían consecuencia de la exposición a distintas claves ambientales en la Sesión II. Se entendió por rehabituación de la RRP al decremento de la misma medido como porcentaje de la amplitud de la RRP en cada ensayo respecto de la amplitud de la RRP en el ensayo 1 de la Sesión II como consecuencia de la exposición repetida al estímulo habituador a lo largo de los ensayos de esta Sesión II. Se tuvo en cuenta también como variable dependiente la recuperación de la respuesta en el primer ensayo de dicha Sesión II. Por tal se entendió el incremento en la amplitud de la RRP en el ensayo 1 de la Sesión II respecto de la amplitud de la RRP en el ensayo 20 de la Sesión I, último de dicha sesión.

\section{Procedimiento}

Tal como se ha indicado los sujetos fueron reclutados dándoseles algunos detalles del experimento. Se les dijo que participarían en un experimento no lesivo en el que no se les pediría hacer nada fuera de sus posibilidades ni se harían comparaciones de capacidad con otros sujetos, la finalidad del experimento era explorar cierto proceso de aprendizaje y se requeriría emplear dos sesiones de treinta minutos aproximadamente, separadas entre sí veinticuatro horas. Si decidían participar debían asistir a ambas sesiones necesariamente. Después de su decisión afirmativa se les dio día, hora y lugar.

En la sesión I, tanto del grupo $E$ como del grupo $C$, cada sujeto fue introducido en una cabina experimental y sentado en una butaca giratoria de brazos, desde la que se podía observar lo siguiente: paredes acorchadas de color blanco, techo del mismo color con una tulipa para tubos fluorescentes, una mesa sobre la que había un bafle marrón a un metro del sujeto, 
un espejo (unidireccional), unas cortinas marrones, un radiador de calefacción y suelo de color gris moteado. En la cabina había un débil olor a tela de saco, una humedad relativa del aire del $50 \%$ y una temperatura de $22{ }^{\circ} \mathrm{C}$. Fuera de la cabina estaban situados los aparatos empleados (salvo el altavoz) y el experimentador.

Una vez sentado cómodamente el sujeto se le colocaron los electrodos para la medida de la RRP en la falange media de los dedos índice y corazón de la mano izquierda, que fue situada reposando sobre el brazo de la butaca. A continuación se les dio las siguientes instrucciones:

"Permanece relajado y no hagas grandes movimientos, sobre todo mantén inmóvil el brazo y la mano izquierda. Lo único que tienes que hacer es estar atento a lo que pueda suceder, que nunca será algo peligroso. Cuando la sesión haya terminado entraré de nuevo aquí para avisarte ¿de acuerdo?»

Tras pasar a la sala de registro, el experimentador registró la RRP durante cuatro minutos en los cuales se observaban las fluctuaciones espontáneas de la RRP con el fin de eliminar a un sujeto anormal en este sentido. De hecho ninguno lo fue y este período de tiempo sirvió para conseguir la estabilización de la respuesta. Una vez transcurrido este tiempo, se les administraron 20 tonos de $100 \mathrm{~Hz}, 105 \mathrm{~dB}, 2,5$ segundos de duración y de onda cuadrada, con un intervalo variable entre estímulos de sesenta segundos de media (rango: 50-70 segundos).

Se registró la RRP desde diez segundos antes hasta quince segundos, al menos, después de cada tono.

Durante la sesión II el grupo C recibió, veinticuatro horas después, el mismo tratamiento que en la sesión I y en la misma cabina experimental, que se encontraba en idénticas condiciones a las del día anterior. El grupo E recibió, también veinticuatro horas después otros veinte tonos de idénticas características pero administrados en una situación diferente: el lugar llamado "Casa Magnética" situado en el Parque de Atracciones de Madrid. Consta de un pequeño pasillo en forma de $\mathrm{L}$ inclinado en su último tramo, por el que se accede a una sala de unos $25 \mathrm{~m}$., inclinada respecto al plano horizontal sobre el vértice de entrada unos $25^{\circ}$. Al entrar en la sala no se aprecia la inclinación al estar todos los objetos inclinados igualmente respecto a la horizontal, de tal manera que al andar hay que inclinar el cuerpo hacia adelante intentando vencer una "fuerza magnética" que atrae hacia el vértice de giro. Unos tubos fluorescentes morados producen una extraña iluminación que hace que resaltar el color blanco y los marcos de las dos ventanas (cerradas) existentes y algunos cuadros pintados con material fosforescente, además, en el centro de la sala hay una mesa y en una de las paredes una chimenea y un gran espejo. Se añadió una silla para realizar el experimento. La temperatura en el interior era de $17^{\circ} \mathrm{C}$, una humedad del $65 \%$, olor a humo de madera quemada y se escuchaba ligeramente el sonido de un motor eléctrico. Lo mismo que en la sesión I, la instrumentación, salvo el altavoz y el sujeto experimental, estaban situados fuera de la sala. Después de realizada la sesión II se les preguntó a los sujetos si habían estado antes en la Casa Magnética y qué les parecía la situación, con objeto de comprobar si realmente era nueva para ellos. (Ya se ha indicado que un sujeto fue eliminado por esta razón.) 


\section{Resultados}

Se calculó la amplitud relativa de la RRP (Respuesta a resistencia de la piel) ante cada estímulo como porcentaje de la amplitud de la respuesta ante la primera presentación del estímulo en cada fase, y su promedio para cada ensayo.

La figura 1 muestra las curvas de habituación del grupo experimental y del grupo de control en la sesión I. Como puede observarse son curvas de habituación típicas, sin ninguna característica especial. Tanto el grupo experimental como el de control alcanzan niveles de habituación compara-

FIGURA 1

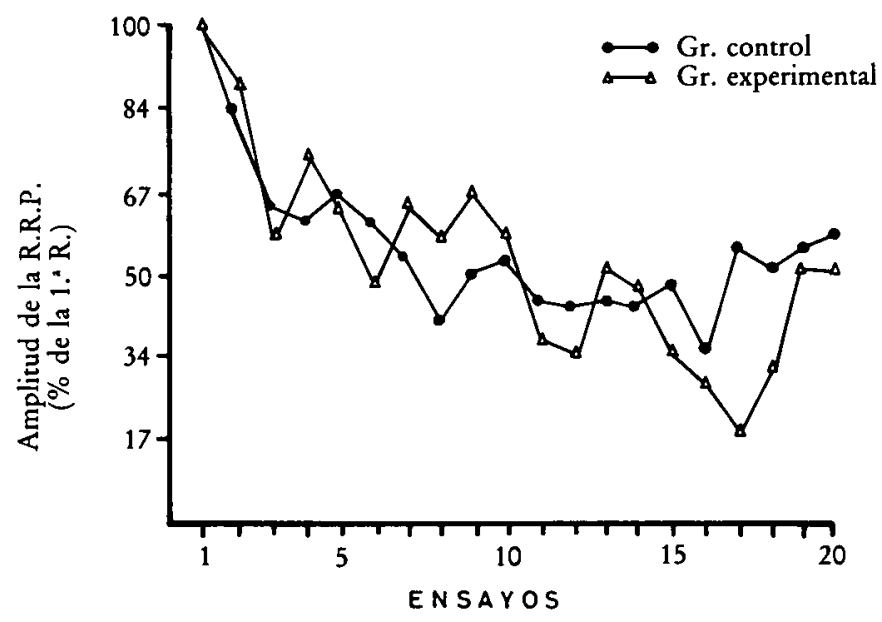

Curvas de habituación del Grupo de Control y del Grupo Experimental en la sesión I

bles a lo largo de los 20 ensayos (y por ello, curvas de habituación también similares) y no existen diferencias significativas entre ellos en reactividad (excitabilidad) en el ensayo I.

La figura 2 muestra las curvas de habituación de los grupos experimental y de control en la sesión II. Se observa una mayor habituación en el grupo $\mathrm{E}$ (claves ambientales nuevas) frente al grupo $\mathrm{C}$ (claves ambientales idénticas), pero estas diferencias sólo son estadísticamente significativas en los ensayos 2 ( $U$ de Mann-Witney $=12 p \leqslant 0,05), 6(U=7, p \leqslant 0,01), 9$ $(U=11, p \leqslant 0,05), 13(U=7, p \leqslant 0,01), 15(U=9, p \leqslant 0,025)$, $16(U=12, p \leqslant 0,05), 17(U=11, p \leqslant 0,05), 18(U=4, p \leqslant 0,01)$, $19(\mathrm{U}=3, \mathrm{p} \leqslant 0,01)$ y $20(\mathrm{U}=9, \mathrm{p} \leqslant 0,025)$.

Se calculó también la recuperación de la respuesta en el ensayo I de la sesión II como diferencia cambiada de signo entre la amplitud de la respuesta en el ensayo 20 de la sesión I y la amplitud (también en relación al ensayo I de la sesión I) de la respuesta en el ensayo 1 de la sesión II. La figura 3 resume los resultados obtenidos en ambos grupos. Puede observarse que el grupo $\mathrm{E}$ (claves ambientales nuevas) tuvo una recuperación de la respuesta en el primer ensayo de la sesión II menor que la del grupo C (claves ambientales idénticas), aunque dicha diferencia no alcanzó por poco el nivel de significación estadística requerido. 
FIGURA 2

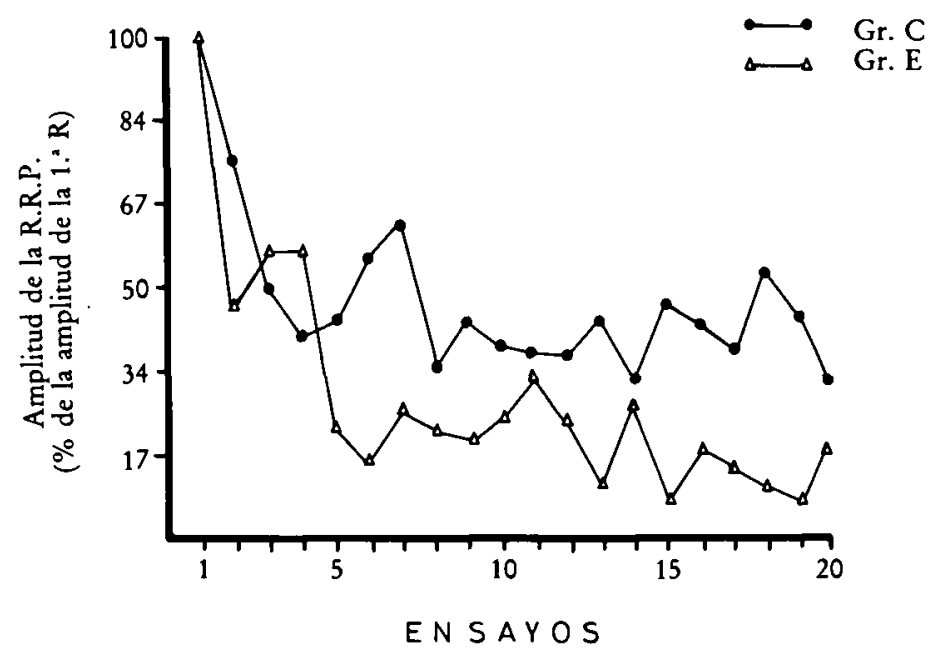

Curvas de habituación del Grupo de Control y del Grupo Experimental en la sesión II

FIGURA 3

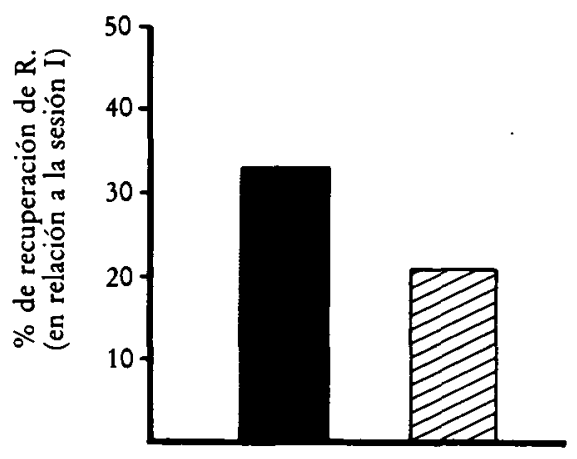

Gr. C. Gr. E.

Recuperación de la respuesta en el ensayo primero de la sesión II

\section{Discusión}

Los resultados encontrados no apoyan al modelo teórico de Wagner $(1976,1978)$ en relación a la habituación a largo plazo. Así la recuperación de la respuesta en el primer ensayo de la sesión II, que debemos considerar como la más directa verificación de dicho modelo de las dos variables dependientes aquí consideradas, no difiere en ambos grupos, es decir, el cambio de contexto no produce una recuperación diferente de la respuesta ya habituada. Los resultados, como se ha mostrado, aunque no alcanzan por poco el nivel de significación estadística, van en la dirección contraria: los sujetos que cambiaron de claves ambientales tendieron a mostrar una menor recuperación de la respuesta que aquellos que volvieron a encontrarse ante el estímulo habituador en la misma situación. 
En cuanto a la rehabituación, los resultados tampoco apoyan al modelo teórico de Wagner, al no producirse una rehabituación más lenta como consecuencia del cambio de contexto. La curva de rehabituación del grupo experimental es, en todo caso, más rápida, siendo más patente esta diferencia a medida que los ensayos de rehabituación progresaron. Una pauta parecida fue encontrada ya por Glez. Almendros (1983) empleando la respuesta de sobresalto en ratas.

Es de resaltar que el cambio de claves ambientales que se ha producido en el grupo experimental en este trabajo es muy considerable, pues la situación experimental de este grupo en la sesión II difería en su localización (los sujetos fueron transportados individualmente a unos kilómetros de distancia del lugar de realización de la sesión 10 y en todas sus claves ambientales restantes. Puede decirse que la única constante, además de la presentación de los tornos, era el hecho de tratarse de una situación de prueba, pero esto, naturalmente, es difícilmente salvable.

En conclusión, los datos de este experimento no permiten confirmar las predicciones hechas por el modelo téorico de Wagner $(1976,1978)$, respecto a la habituación con las claves ambientales $y$, más bien, van en la dirección contraria, al menos en algunos aspectos. Creemos que con un mayor número de sujetos por grupo y quizá tratando de disminuir la variabilidad de los mismos mediante unas instrucciones menos vagas, posiblemente se encontrarian diferencias significativas en la recuperación de las respuestas en el ensayo 1 de la sesión II, con lo que se confirmaría la tendencia contraria a la predicha por el modelo de Wagner hallada en este experimento. Además, las instrucciones pueden emplearse también para conseguir que el estímulo habituador sea más predictible, con lo que la habituación debería ser también más rápida según dicho modelo.

\section{EXPERIMENTO 2}

En este segundo experimento se pretendía también verificar el papel que la supuesta asociación entre el estímulo habituador y las claves ambientales tuviera en la habituación a largo plazo. Para ello se manipularán estas claves ambientales entre una y otra sesión siguiendo la estrategia del experimento 1; además, se manipularon también las instrucciones administradas a los sujetos. Se suponía que si, tal como Wagner $(1976,1978)$ afirma, la habituación se debe a que el estímulo habituador deja de ser sorpresivo, entonces si se dan instrucciones concretas a los sujetos acerca de lo que va a suceder en el experimento y concretamente del estímulo que va a aparecer, tal estímulo será menos sorpresivo y producirá un decremento más rápido en la respuesta por él elielitada.

Debido a que el tono empleado como estímulo en el experimento 1 era de comienzo súbito y de intensidad moderada-alta $(105 \mathrm{~dB})$ pudiera ser que, en vez de provocar una respuesta de orientación produjera una respuesta de defensa y, ya que estos tipos de respuesta tienen una habituación diferente (Sokolov, 1963), en este segundo experimento se empleó un tono de menor intensidad y de comienzo gradual para no provocar sobresalto (una respuesta defensiva).

Pudiera ser también que los resultados negativos respecto al modelo teó- 
rico de Wagner se debieran a que la habituación a largo plazo no fuese manifiesta al cabo de veinticuatro horas, posibilidad remota. Por ello, en este experimento segundo se empleó un intervalo entre sesiones de cuarenta y ocho horas en vez de veinticuatro como en el anterior. Se incrementó también el número de sujetos por grupo.

\section{Método}

\section{Sujetos}

Se emplearon 40 sujetos: 18 varones y 20 hembras, todos ellos estudiantes del tercer curso de BUP, alumnos de un mismo centro de enseñanza, el Instituto Eijo y Garay, con edades comprendidas entre 16 y 19 años con una media de 16,8; todos fueron participantes voluntarios después de explicarles en una breve charla la necesidad de unos sujetos para la realización de un experimento de psicología. Dos de estos sujetos fueron eliminados al no dar ninguna respuesta en la sesión I.

\section{Aparatos}

El estímulo auditivo fue un tono de $400 \mathrm{hz}$, cuya presentación se realizaba de forma gradual desde $0 \mathrm{db}$ a $80 \mathrm{db}$ con una duración de tres segundos. Se grabó una cinta magnetofónica con 20 tonos de este tipo, con un intervalo entre ellos de sesenta segundos (rango 51-69), que era reproducida por un magnetófono estereofónico Sanyo. La respuesta de resistencia de la piel (RRP) se registró mediante un polígrafo Lafayette LA-76100-01. Para identificar la respuesta al tono se midió la amplitud de la RRP entendida como la máxima deflexión de la curva cuyo inicio ocurría entre uno y cuatro segundos después del inicio de dicho tono.

\section{Diseño}

Se empleó un diseño factorial $2 \times 2$. Los sujetos fueron divididos al azar en cuatro grupos que recibieron entrenamiento en habituación en dos sesiones (sesión I y sesión II) separadas entre sí cuarenta y ocho horas. Como variables independientes se utilizaron dos situaciones ambientales diferentes (claves ambientales I y claves ambientales $P$ ) y dos tipos de instrucciones: instrucciones neutras (A), que no indicaban al sujeto lo que iba a suceder pero tampoco se les instruía en alguna actividad distractiva, e instrucciones informativas (B) por las que los sujetos recibían una información detallada tanto del estímulo como del procedimiento y propósito del experimento (ver anexo 1). El tratamiento de cada grupo está recogido en la tabla II.

Los sujetos de los grupos 1 y 2 recibieron el entrenamiento en la sesión I y II en presencia de distintas claves ambientales. El grupo 1 recibió instrucciones neutras y el grupo 2 instrucciones informativas; los sujetos de los grupos 3 y 4 recibieron el entrenamiento en el mismo ambiente en ambas sesiones; el grupo 3 con instrucciones neutras y el grupo 4 con instrucciones informativas.

Se utilizaron como variables dependientes la habituación y rehabituación de la RRP consideradas como el decremento de la misma medido como 
TABLA II

Diseño empleado en el experimento II

\begin{tabular}{c|cc|cc|}
\multicolumn{1}{c}{} & \multicolumn{3}{c}{ Sesión I } & \multicolumn{3}{c}{ Sesión II } \\
\cline { 2 - 5 } Grupo 1 & Claves ambientales & I & Claves ambientales & P \\
& Instrucciones & A & Instrucciones & A \\
\cline { 2 - 5 } Grupo 2 & Claves ambientales & I & Claves ambientales & P \\
Grupo 3 & Instrucciones & B & Instrucciones & B \\
\cline { 2 - 5 } & Claves ambientales & I & Claves ambientales & I \\
Grupo 4 & Instrucciones & A & Instrucciones & A \\
\cline { 2 - 5 } & Claves ambientales & I & Claves ambientales & I \\
\cline { 2 - 5 } & Instrucciones & B & Instrucciones & B \\
\hline
\end{tabular}

porcentaje de la amplitud de la RRP en cada ensayo respecto de la amplitud de la RRP en el ensayo 1 de la sesión I, la habituación y en el ensayo 1 de la sesión II, la rehabituación, como consecuencia de la repetida presentación del estímulo habituador.

Para estudiar las influencias que el cambio de claves ambientales tienen sobre la habituación, se unieron los grupos 1 y 2 y se observaron las diferencias con los grupos 3 y 4 , de tal manera que el grupo 1,2 estaba formado por 19 sujetos, nueve de los cuales habían recibido instrucciones $\mathrm{A}$ y 10 instrucciones B. Por tanto, diferían solamente en el entrenamiento recibido en la sesión II; el grupo 1,2 lo había hecho con las claves iguales que en la sesión I, de tal manera que las diferencias en la rehabituación serían consecuencia de la exposición a diferentes claves.

Igualmente para estudiar el efecto de las instrucciones se agruparon los grupos 1 y 3 , ambos instruidos con instrucciones neutras y los grupos 2 y 4 que recibieron instrucciones informativas. El grupo 1,3 estaba formado por 18 sujetos, nueve habían recibido el entrenamiento en presencia de diferentes claves ambientales en sesión I y sesión II y nueve lo habían recibido en el mismo ambiente. El grupo 2,4 estaba formado igualmente por 19 sujetos, diez habían recibido entrenamiento en las mismas claves en ambas sesiones, y diez lo habían recibido en claves diferentes.

También se consideraron como variables dependientes la tasa de habituación, que se entendió como el número de ensayos transcurridos hasta el primero de ellos en que el sujeto no dio respuesta y la recuperación de la respuesta en el ensayo 1 de la sesión II, que se entendió como la diferencia entre la amplitud de la respuesta en el esnayo 20 de la sesión I (en relación al ensayo 1 de la sesión I) y la amplitud de la respuesta en el ensayo 1 de la sesión II (en relación también al ensayo 1 de la sesión I).

\section{Procedimiento}

Los sujetos utilizados se inscribían voluntariamente después de dar una breve charla en sus aulas en la que se comentaban algunos aspectos generales de la psicología, la necesidad de la investigación, etc., por último, se pedía su colaboración para un experimento en el que se trataba de explorar 
las posibles utilizaciones de un aparato. Se les informó que el experimento constaba de dos pruebas diferentes con dos días de intervalo entre ellas.

En la sesión I los sujetos accedían al lugar de experimentación individualmente; allí se les explicaba el funcionamiento del polígrafo, y se les daban las instrucciones que correspondieran, los grupos 1 y 3 recibieron las instrucciones neutras (A) en las que se les explicaba que el experimento consistía principalmente en probar el aparato, cuyas posibilidades no eran del todo conocidas, para posteriormente poder hacer un completo uso de él. Los grupos 2 y 4 recibieron unas instrucciones informativas (B) en las cuales se les daba información detallada del aparato y su utilización así como del procedimiento y propósito del experimento. Se les daba detalles de las características de los tonos, se les comunicaba el número de tonos, el intervalo entre ellos y su duración. Inmediatamente después pásaban a la sala experimental que denominamos claves ambientales I y que consisten en un camerino anexo al escenario del salón de actos del Instituto, de unos $10 \mathrm{~m}$., techo y paredes de color blanco, iluminado por luz de día y dos tubos fluorescentes; la luz de día entraba por un amplio ventanal de una de las paredes, una persiana impedía que se viera al exterior. En el interior de esta sala había unas puertas que daban acceso a un armario empotrado, un mueble alto, una pequeña mesa a la espalda del sujeto donde se situaba el altavoż y dos sillas. Fuera de la sala y cercano a la puerta de acceso se encontraban los aparatos empleados y el experimentador.

Una vez dentro los sujetos se sentaban en una de estas sillas y se les instruía en permanecer lo más quietos y relajados posible, aconsejándoles que adquirieran una posición cómoda. Les eran colocados los electrodos en la falange media de los dedos índice y medio de la mano derecha. La mano era colocada sobre la pierna derecha de los sujetos en una posición cómoda. Se les anunciaba que al final del experimento serían avisados y se les dejaba cinco minutos antes de iniciar la presentación de los tonos.

La sesión II transcurrió en la misma forma cuarenta y ocho horas después; los sujetos de los grupos 1 y 2 realizaban esta sesión en una sala experimental distinta, que denominamos claves ambientales $\mathrm{P}$, de características similares a las descritas en el experimento 1 (Casa Magnética), exceptuando el olor a madera quemada que no existía y añadiendo un sonido de fondo producido por un extractor de humnos. El tiempo total de duración de cada sesión era de treinta minutos; después de cada una de ellas los sujetos rellenaban un cuestionario en el que se indagaba sobre su idea del experimento, como creían que debían actuar, etc. El modelo aparece en el anexo 2 .

\section{Resultados}

En la figura 4 se ha representado las curvas de habituación de los cuatro grupos en la sesión I; se observan curvas similares para los cuatro grupos no encontrándose diferencias significativas entre ninguno de ellos a lo largo de las mismas.

Las figuras 5 y 6 muestran las curvas de rehabituación (sesión II). No se aprecian diferencias significativas entre los grupos con cambio contextual (1 y 2) y los grupos sin cambio contextual (3 y 4), ni entre los grupos con instrucciones neutras $\left(\begin{array}{lll}1 & \text { y } 3\end{array}\right)$ y los grupos con instrucciones informativas $(2$ y 4$)$. 
Figura 4

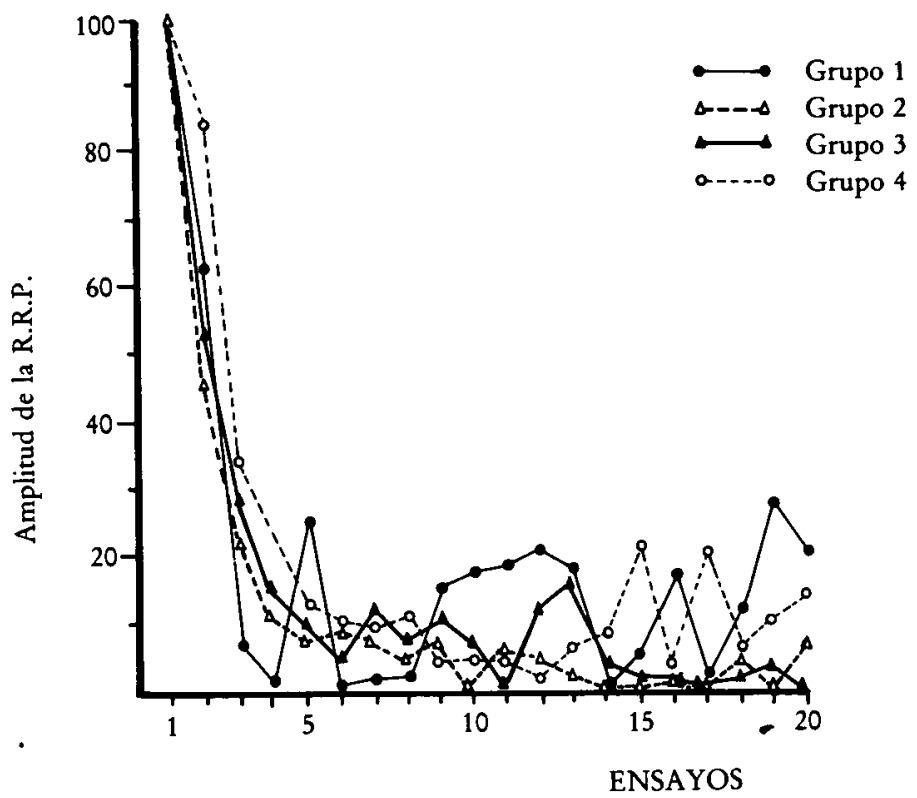

Curvas de babituación de los cuatro grupos en la sesión I

En cuanto a la tasa de habituación (tabla III) tampoco se han encontrado diferencias entre los grupos de instrucciones neutras ( 1 y 3 ) y los de instrucciones informativas ( 2 y 4) ni en la sesión I ni en la sesión II; sin embargo, para los grupos con cambio contextual (1 y 2) y los grupos sin cambio contextual ( 3 y 4 ) se ha encontrado una diferencia a favor de los primeros en la sesión II, aunque a un bajo nivel de significación ( $T,-1,36$, $\mathrm{p} \leqslant 0,1)$. En la sesión I tampoco se encontraron diferencias.

En la figura 7 se muestra la recuperación de la respuesta en el ensayo 1 de la sesión II, se aprecia una mayor recuperación en los grupos sin cambio ambiental ( 3 y 4) significativamente mayor que la de los grupos con cambios de claves entre sesiones (1 y 2) ( $U$ de Mann-Witney ,35, $p \leqslant 0,001$ ). También se aprecia una recuperación menor para los grupos de instrucciones informativas frente a los grupos de instrucciones neutras, sin embargo la diferencia no alcanza el nivel de significación requerido.

\section{Discusión}

Los resultados de este experimento muestran en primer lugar que el cambio en las claves ambientales no produce los efectos esperados de acuerdo con el modelo teórico de Wagner $(1976,1978)$, que son, en algún aspecto, incluso contrarios, $y$, en segundo lugar, que las instrucciones tampoco producen el efecto esperado en este sentido.

Respecto al efecto de las claves ambientales, las curvas de rehabituación (sesión II) son ligeramente más rápidas en el grupo expuesto a un cambio en las claves ambientales entre una y otra sesión de habituación frente al que recibió ambas sesiones en el mismo ambiente. Este resultado es de sen- 
tido opuesto a lo esperado según el modelo de Wagner, pero concuerda con los resultados encontrados en el Experimento I, en humanos, y por Glez. Almendros (1983), en ratas. El efecto, sin embargo, es muy ligero y no alcanza una gran significación; un resultado similar, en el sentido de que la tasa de habituación no muestre diferencias, ha sido encontrado en otras investigaciones tanto en animales (Leaton 1974, Marlin y Miller 1981) como en humanos (Averrill y cols. 1972).

FIGURA 5

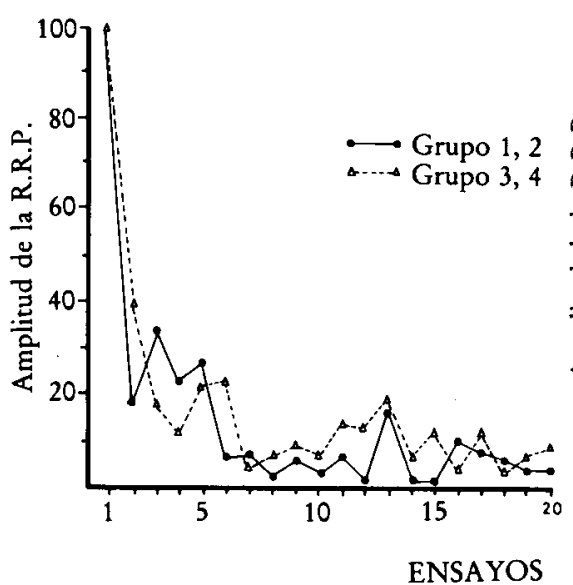

Curvas de rehabituación teniendo en cuenta el efecto del cambio de contexto
FIGURA 6

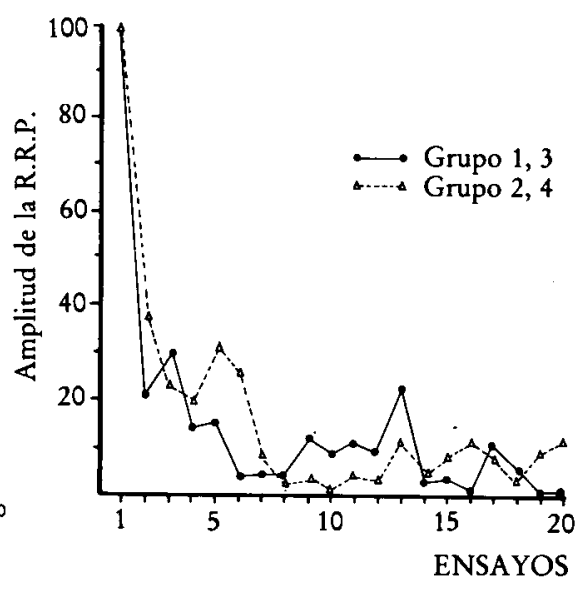

Curvas de rebabituación teniendo en cuenta los efectos de los tipos de instrucción.

\section{TABLA III}

Tasas de habituación teniendo en cuenta el cambio de contexto o las instrucciones: con cambio contextual (grupos 1 y 2), sin cambio contextual (grupos 3 y 4); instrucciones neutras (grupos 1 y 3) $e$ instrucciones informativas (grupos 2 y 4).

\begin{tabular}{|c|c|c|}
\hline & Sesión I & Sesión II \\
\hline Grupo 1, 2 & 2,71 & 1,78 \\
\hline Grupo 3, 4 & 3,38 & 2,84 \\
\hline Grupo 1, 3 & 2,44 & 2,27 \\
\hline Grupo 2, 4 & 3,04 & 2,35 \\
\hline
\end{tabular}

Tasas de babituación.

La recuperación de la respuesta en el primer ensayo de la sesión II, que, como se ha señalado, es la variable dependiente más válida para comprobar el modelo de Wagner al hacer un cambio en las claves ambientales entre sesiones y sobre todo en este experimento en el que la habituación muy rápida, tampoco apoya dicho modelo. Al contrario, aquí los resultados son claramente opuestos a los previstos: los sujetos que recibieron el entrenamiento en habituación en presencia de las mismas claves ambientales en ambas sesiones se mostraron una recuperación de la respuesta en el ensayo 1 


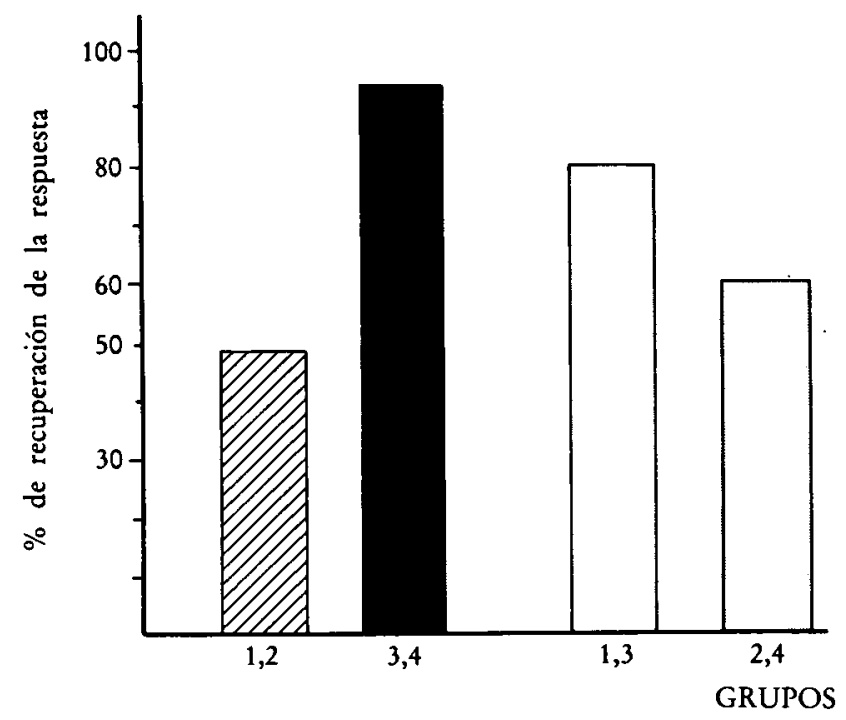

Recuperación de la respuesta en el ensayo primero de la sesión II teniendo en cuenta el cambio de contexto o las instrucciones; con cambio contextual (grupos 1 y 2), sin cambio contextual (grupos 3 y 4); instrucciones neutras (grupos 1 y 3) $e$ instrucciones informativas (grupos 2 y 4).

de la sesión II mucho mayor (casi el doble) que aquellos que recibieron esta sesión II ante claves completamente nuevas. Esto confirma los resultados en esta dirección, pero que no alcanzaban el nivel de significación deseado, encontrados en el experimento I.

Respecto a las instrucciones, no se observa efecto alguno de las mismas sobre las variables dependientes utilizadas (habituación, rehabituación, tasa de habituación y recuperación de la respuesta) en contra de lo esperable. Estos resultados concuerdan con los de Iacono y Lykken (1983), quienes no encontraron diferencias entre instrucciones de "atender" al estímulo e instrucciones informativas. Otras investigaciones, sin embargo, han obtenido resultados contradictorios empleando distintos tipos de instrucciones (Harding y Punzo, 1971; Barry, 1977; Maltzman y Raskin, 1979).

En resumen, tanto los efectos del cambio en las claves ambientales como los de las instrucciones no apoyan al modelo teórico de Wagner (1976, 1978) para la habituación a largo plazo. Mientras las instrucciones informativas no producen ningún efecto, el cambio en las claves ambientales nuevas atraerían la atención de los sujetos en la segunda sesión cuando ésta se realiza en un ambiente diferente. Debido a nuestra limitada capacidad de procesamiento atencional que hace que nuestra atención sea selectiva (véase p. e., Lindsay y Norman, 1977), tal atención a las claves ambientales nuevas ha de ir en detrimento de la atención al estímulo habituador, lo que contribuiría al proceso de habituación entendido como un proceso mediante el cual el organismo aprende a ignorar aquellos estímulos que mantienen características constantes en sus presentaciones sucesivas. Tal interpretación se incluye dentro de una consideración atencional del procesamiento de un estímulo. Así, Berlyne (1966) hizo algunas consideraciones sobre los estí- 
mulos "prosexígenos" como aquellos cuyas características (imprevistos, insólitos, hetereogéneos, asimétricos, incoherentes y complejos) provocan una elevación del nivel de vigilancia. Otros autores (Brown y cols., 1977; Ohman, 1979) han considerado la importancia del significado de los estímulos como determinante de la atención (los estímulos atendidos pasan a ser procesados por el canal central de capacidad limitada), en relación con el reflejo de orientación.

\section{CONCLUSIONES}

Los resultados negativos encontrados por otros investigadores respecto al papel que las claves ambientales desempeñan en la habituación a largo plazo han sido, en general, aquí confirmados respecto a sujetos humanos sometidos a habituación acústica de la Respuesta de Resistencia de la Piel con sonidos puros, tanto de comienzo súbito como gradual, tanto con instrucciones «neutras" como con instrucciones "informativas» $y$ tanto con un período de retención de veinticuatro como de cuarenta y ocho horas. Quizás la excepción más destacable haya sido la menor recuperación de la respuesta que se observa en el primer ensayo de la segunda sesión cuando ésta se lleva a cabo ante claves ambientales diferentes de las de primera.

Respecto a la validez del modelo teórico de Wagner $(1976,1978)$, hemos de decir que estos resultados se suman a los ya citados en la introducción en los que no se ha encontrado confirmación al mismo. En algunos casos, los efectos encontrados son de sentido opuesto a lo esperable, como el mencionado respecto a la recuperación de la respuesta o la tasa de habituación en la sesión II. Creemos que se ha acumulado suficiente información para empezar a sospechar que dicho modelo no se ajusta en absoluto a la realidad del proceso de habituación, al menos de la habituación a largo plazo. Existen algunas excepciones que merecen un estudio más detallado, pero, en general, los datos nos indican que la habituación se parece más a la idea tradicional de un proceso de aprendizaje relativamente simple fundamentalmente dependiente de las características del estímulo habituador. El experimento II parece resaltar la importancia que en este proceso parece tener la atención y sus resultados más bien concuerdan con la tradicional consideración de la habituación como un proceso de aprender a ignorar un estímulo reiterativo.

Esto no significa que el contexto en el que se presente el estímulo habituador no influya en la habituación. De hecho, se ha observado que el cambio de claves ambientales produce una recuperación de la respuesta menor y una tendencia a una mayor tasa de habituación. Además del contexto físico, creemos que, puesto que la habituación es un proceso central en el que el cortex juega un papel decisivo (Sokolov, 1963), quizás sea más dependiente de cambios «psicológicos" que el modelo de Wagner no considera y que no han sido verificados sistemáticamente hasta el momento. Sin embargo, sirvan nada más como ejemplo de que tal posibilidad es probable los resultados de Luria y Vinogradova (1959) y Maltzman y Raskin (1979), quienes han mostrado cómo la tarea que el sujeto debe realizar durante el entrenamiento en habituación influye en esta última. Puede decirse que el sujeto se encuentra, en esos, ante situaciones diferentes; en este sentido, el 
trabajo de Luria y Vinogradova es el más relevador, pues tal efecto se produjo cuando el sujeto pasaba sucesivamente de una a otra tarea, es decir, al cambiar de «situación psicológica».

\section{Referencias}

Averrill, J. R.; Malmstronm, E. J.; Koriat, A., y Lazarus, R. S. (1972). Habituation to complex emotional stimuli. Journal of abnormal Psychology, 30, $20-28$.

BARRY, R. J. (1977). The effect "significance" upon indices of Sokolov' orienting responses. Anex conceptualization to replace the O. R. Physiological Psychology, S, 209-214.

BerLyNe, D. E. (1966). Conflict and arousal. Scientific American, 215, 82-87.

Brown, J. W.; MORSE, P. A., y Grahan, F. K. (1977). Response to auditory stimuli in 6 and 9 week-old humans infants. Development Psychology, 18, 189-196.

Glez. Almendros, J. L. (1983). Claves internas y externas en la habituación a largo plazo. Anuario de Psicología, 28, 49-58.

HARDING, G., y PUNZO, F. (1971). Response uncertainty and skin conductance. Journal of Experimental Psychology, 88, 265-272.

LEATON, R. N. (1974). Long-term retention of the habituation of Lick suppression in rats. Journal of Comparative Physiological Psychology, 87, 1157-1164.

LINDSAY, P. H., y NORMAN, D. A. (1977). Human information processing: an introduction to psychology. Nueva York: Academic Press. [Trad. cast.]: Introducción a la psicologia cognitiva. Madrid: Tecnos, 1983.

LURIA, A. R., y VINOGRADOVA, O. S. (1959). An objetive investigation of the dynamics of semantic systems. British Journal of Psychology, 30, 89-105.

MALTZMAN, I., y RASKIN, D. C. (1979). Selective orienting and habituation of the GSR as a consecuence of overt and covert activity. Physiological Psbychology, 7, 204-208.

MARLIN, N. A., y MiLLER, R. R. (1981). Associattion to contextual stimuli as a determinant of Long-term habituation. Joumal of Experimental Psychology: Animal Behavior Processes, 7, 313-333.

OhMAN, A. (1979). The orienting response, attention and learning: an information-processing perspective. En H. D. Kimmel; E. H. Van Olst y J. F. Orlebeke (comps.): The Orienting reflex in humans. Hillsdale, N. J.: Erlbaum Associates.

PEEKE, H. V. S., y VENO, G. (1973). Stimulus specificity of habituated aggression in three'spined sticklebacks. Behavior Biology, 8, 427-432.

Sokolov, R. O. (1963). Percepcion and the conditioned reflex. Oxford: Pergamon Press.

WAGNER, A. R. (1976). Priming in STM: An information-processing mechanism for self-generated or retrieval-generated depression in performance. En T. J. Tighe y R. N. Leaton (comps.): Habituation: Perspective from child development. Animal behavior and neurophysiology. Hillsdale, N. J.: Erlbaum Associates.

WAGNER, A. R. (1978). Expectances and the priming of STM. En S. H. Hulse, H. Fowler y W. K. Honig (comps.): Cognitive processes in animal behavior. Hillsdale, N. J.: Erlbaum Associates.

\section{Anexo 1}

\section{INSTRUCCIONES A (NEUTRAS)}

Este aparato que ves aquí es un polígrafo (se les enseñaba el aparato, se conecta y se mueve la aguja para que aprecien los registros). Estamos intentando descubrir sus posibles utilidades; para ello estamos realizando una serie de experimentos como éste en el que tú vas a participar. Tu misión va a ser muy sencilla, se trata de que te sientes en esta silla (se le enseña la silla) en una posición lo más cómoda que te sea posible. Yo te pondré unos electrodos en los dedos para que el polígrafo recoja la señal y podamos apreciar su funcionamiento en esta situación. ¿ De acuerdo? Es importante que permanezcas tranquilo y lo más relajado que puedas. Procura no moverte, 
pero sobre todo procura no mover la mano donde tienes los electrodos. Los movimientos pueden alterar el funcionamiento del polígrafo. ¿Estás dispuesto a empezar?

\section{INSTRUCCIONES B (INFORMATIVAS)}

Este aparato que ves aquí se llama polígrafo y registra las señales procedentes de estos electrodos que luego te colocaré. (Se le enseña el aparato, se conecta y se mueve la aguja para que vean el registro.) Las señales que registra son las que le manda tu organismo. Tu misión es sentarte en esa silla (se le enseña la silla) en una posición lo más cómoda posible. Yo te pondré estos electrodos. Inmediatamente después dará comienzo el experimento. Pasarán cinco minutos sin que suceda nada, después sonará un pitido que se repetirá 20 veces, este pitido es de $400 \mathrm{~Hz}$ de frecuencia, para que te hagas una idea, el pitido de la señal horaria que habrás oído alguna vez es de $1.000 \mathrm{~Hz}$, es decir, que el que vas a oír es más grave. Los pitidos empezarán a una intensidad $0 \mathrm{~dB}$ e irán aumentando hasta $80 \mathrm{~dB}$. A este máximo volumen el pitido es menor que el producido por las bocinas de los coches. Cada pitido durará tres segundos y entre uno y otro pasarán unos sesenta segundos, aunque algunos tardarán algo más y otros algo menos.

Es importante que atiendas a los tonos. Tu cuerpo reaccionará cada vez que suene y esa reacción es la que registrará el polígrafo. Probablemente después de unos cuantos pitidos tu organismo dejará de reaccionar, eso es que se habrá habituado.

Debes sentarte en una posición cómoda y relajada, e intentar no moverte, sobre todo debes evitar mover la mano donde están colocados los electrodos. La sesión durará treinta minutos. Después de que suene el último tono te avisaremos. ¿Estás dispuesto a empezar?

\section{Anexo 2}

\section{CUESTIONARIO:}

\section{NOMBRE:}

1. ¿Tenías alguna idea preconcebida antes de realizar el experimento? ¿Qué pensabas que iba a ocurrir?

2. Después de haber escuchado las instrucciones, ¿tenías claro la forma en que debías actuar correctamente?

3. ¿En qué consistía para ti actuar correctamente?

4. ¿Sabías en qué consistía el experimento, después de las explicaciones recibidas?

5. ¿En qué momento te diste cuenta que sólo era un sonido intrascendente que se repetía de vez en cuando?

6. ¿Qué pensabas durante el experimento? 